\title{
Strangeness of the nucleon from lattice QCD
}

\author{
Constantia Alexandrou, ${ }^{1,2}$ Martha Constantinou, ${ }^{2}$ Simon Dinter, ${ }^{4}$ Vincent Drach, ${ }^{3,4}$ Kyriakos Hadjiyiannakou, ${ }^{2}$ \\ Karl Jansen, ${ }^{2,4}$ Giannis Koutsou, ${ }^{1}$ and Alejandro Vaquero ${ }^{1}$ \\ (ETM Collaboration) \\ ${ }^{1}$ Computation-based Science and Technology Research Center (CaSToRC), The Cyprus Institute, \\ 20 Constantinou Kavafi Street, Nicosia 2121, Cyprus \\ ${ }^{2}$ Department of Physics, University of Cyprus, P.O. Box 20537, 1678 Nicosia, Cyprus \\ ${ }^{3}$ CP3-Origins \& the Danish IAS, University of Southern Denmark, \\ Campusvej 55, DK-5230 Odense M, Denmark \\ ${ }^{4}$ NIC, DESY, Platanenallee 6, D-15738 Zeuthen, Germany \\ (Received 7 October 2013; revised manuscript received 25 February 2015; published 8 May 2015)
}

\begin{abstract}
We present a nonperturbative calculation of the strangeness of the nucleon $y_{N}$ within the framework of lattice QCD. This observable is known to be an important cornerstone to interpret results from direct dark matter detection experiments. We perform a lattice computation for $y_{N}$ with an analysis of systematic effects originating from discretization, finite size, chiral extrapolation and excited state effects leading to the value of $y_{N}=0.173(50)$. The rather large uncertainty of this value of $y_{N}$ is dominated by systematic uncertainties which we are able to quantify in this work.
\end{abstract}

DOI: 10.1103/PhysRevD.91.094503

PACS numbers: 12.38.Gc, 14.20.Dh

\section{INTRODUCTION}

The question of the exact composition of the nucleon, i.e. what are the different quark contents of the proton and neutron, is a long-standing problem (see for instance [1]) which can be addressed only through nonperturbative methods. In this paper, we will resort to lattice QCD techniques to address the calculation of the strange quark content of the nucleon which is important to know not only for addressing the fundamental question of the nucleon composition, but also because it plays a most important role in the search for dark matter, as will be discussed in more detail below.

A very useful measure for the strange quark content is the $y_{N}$ parameter,

$$
y_{N} \equiv \frac{2\langle N|\bar{s} s| N\rangle}{\langle N|\bar{u} u+\bar{d} d| N\rangle},
$$

where $u, d$ and $s$ denote respectively the up, down and strange quark fields. We will refer to $y_{N}$ as the strangeness of the nucleon in the following.

As mentioned above, the $y_{N}$ parameter plays an important role in the context of dark matter searches. Experiments which aim at a direct detection of dark matter [2-6] are based on measuring the recoil energy of a nucleon hit by a dark matter candidate. Even if in these processes a dark matter particle would not be detected directly, such experiments allow us to provide bounds on the nucleon dark matter cross section which can in turn be translated into constraints on models of new physics. In many supersymmetric scenarios [7] and in some Kaluza-Klein extensions of the standard model $[8,9]$ the dark matter nucleon interaction is mediated through a Higgs boson. In such a case the theoretical expression of the spin independent scattering amplitude at zero momentum transfer involves the $y_{N}$ parameter. In fact, even rather small changes of the poorly known value of $y_{N}$ can be responsible for a variation of 1 order of magnitude of the nucleon dark matter cross section. Having a better determination of the $y_{N}$ parameter would thus provide better estimates on the size of the cross section or more reliable constraints on dark matter models.

The $y_{N}$ parameter is related to the ratio of the pionnucleon $\left(\sigma_{\pi N}\right)$ and the flavor nonsinglet $\left(\sigma_{0}\right) \sigma$ terms, defined as

$$
\begin{gathered}
\sigma_{\pi N} \equiv m_{l}\langle N|\bar{u} u+\bar{d} d| N\rangle, \quad \sigma_{s} \equiv m_{s}\langle N|\bar{s} s| N\rangle \\
\sigma_{0} \equiv m_{l}\langle N|\bar{u} u+\bar{d} d-2 \bar{s} s| N\rangle
\end{gathered}
$$

where $m_{l}$ denotes the average up and down quark mass, and $m_{s}$ the strange quark mass and where we also introduced to the strange $\sigma$ term $\sigma_{s}$. The $\sigma$ terms $\sigma_{\pi N}$ and $\sigma_{0}$ can be estimated within the framework of chiral effective field theories and using the relation

$$
y_{N}=1-\frac{\sigma_{0}}{\sigma_{\pi N}}
$$

estimates of $y_{N}$ can also be provided.

To be more specific, the value of $\sigma_{\pi N}$ can be extracted from the pion-nucleon cross section data at an unphysical kinematics, known as the Cheng-Dashen point. Values for 
$\sigma_{\pi N}$ extracted in this way read $\sigma_{\pi N}=45 \pm 8 \mathrm{MeV}$ from Ref. [10] (GLS) and $\sigma_{\pi N}=64 \pm 7 \mathrm{MeV}$ from Ref. [11] (GWU). A more recent result has been obtained in Ref. [12] (AMO) which gives $\sigma_{\pi N}=59 \pm 7 \mathrm{MeV}$ [12]. The value of $\sigma_{0}$ can be computed analyzing the breaking of $S U(3)$ in the spectrum of the octet of baryons. Following this strategy an estimate for this quantity is given e.g. in [13] and reads $\sigma_{0}^{\mathrm{I}}=36 \pm 7 \mathrm{MeV}$. A more recent calculation using an improved method based on Lorentz covariant chiral perturbation theory with explicit decuplet-baryon resonance fields suggests $\sigma_{0}^{\mathrm{II}}=58 \pm 8 \mathrm{MeV}$ [14].

Using the aforementioned values for $\sigma_{\pi N}$ and $\sigma_{0}^{\mathrm{I}}$ we obtain the following phenomenological estimates of the $y_{N}$ parameter:

$$
\begin{aligned}
y_{N}^{\mathrm{I}, \mathrm{GLS}} & =0.20(21), \quad y_{N}^{\mathrm{I}, \mathrm{GWU}}=0.44(13), \\
y_{N}^{\mathrm{I}, \mathrm{AMO}} & =0.39(14) .
\end{aligned}
$$

Using $\sigma_{0}^{\mathrm{II}}$ we obtain

$$
\begin{aligned}
y_{N}^{\text {II,GLS }} & =-0.29(29), \quad y_{N}^{\text {II,GWU }}=0.09(16), \\
y_{N}^{\text {II,AMO }} & =0.02(17) .
\end{aligned}
$$

Note that these values from effective field theory (EFT) and phenomenology are affected by substantial errors leading to correspondingly large uncertainties for the cross section for dark matter detection [15].

In this paper, we present a first principle computation of the strangeness of the nucleon using lattice QCD techniques. The difficulty of such a computation for a long time has been that, due to the appearance of disconnected, singlet contributions, the error for $y_{N}$ has been very large. Consequently, it has not been possible to obtain a precise enough value which can be used for calculating the cross section reliably, see later in this manuscript for a discussion of various lattice computations.

In Ref. [16] we were able to make a significant step forward by using a setup of maximally twisted mass fermions which avoids any mixing in the renormalization of the $\sigma$ terms and hence $y_{N}$ does not need to be renormalized. In addition, by employing special noise reduction techniques, amenable for our setup, we could achieve a significant improvement in the signal to noise ratio for $y_{N}$. The shortcoming of our result in Ref. [16] has been that, being a feasibility study only, $y_{N}$ was obtained at only one value of the lattice spacing, a single finite volume and only one quark mass.

Here we want to extend the calculation of Ref. [16] by using different lattice spacings, finite volumes and quark masses such that we can probe the effects of the discretization, the finite volume and nonphysical quark masses. In addition, we now have available a high statistics analysis of excited state effects which, as we will see below, are potentially very dangerous for the computations of $y_{N}$.
Being able to address the systematic uncertainties appearing in a lattice calculation of $y_{N}$, we believe that our computation can provide a reasonable estimate of the $y_{N}$ parameter based on QCD alone and not resorting to effective field theories.

\section{LATTICE QCD CALCULATION}

In our computation of $y_{N}$ we use gluon field configurations generated by the European Twisted Mass Collaboration (ETMC) [17] employing maximally twisted mass fermions. In particular, the setup used here includes a mass-degenerate light up and down quark doublet as well as a strange-charm quark pair, a situation which we refer to as the $N_{f}=2+$ $1+1$ setup. In our analysis we have used two values of the lattice spacing, $a=0.082 \mathrm{fm}$ and $a=0.064 \mathrm{fm}$, to examine lattice cutoff effects. We have a number of light quark masses leading to pseudoscalar meson masses $m_{\mathrm{PS}}$ covering the range from $490 \mathrm{MeV}$ to $220 \mathrm{MeV}$. This mass range allows us to perform the chiral limit with $m_{\mathrm{PS}}$ approaching the physical pion mass $m_{\pi}$. We finally remark that we use a mixed action setup with Osterwalder-Seiler quarks in the heavy quark sector which avoids any mixing due to the isospin violation otherwise occurring in the twisted mass sea quark action. This mixed action still enjoys the automatic $O(a)$ improvement of twisted mass fermions.

The basic quantity needed for the evaluation of $y_{N}$ is the ratio of correlation functions

$$
R\left(t, t_{s}\right) \equiv \frac{\sum_{\mathbf{x}_{\mathbf{s}}, \mathbf{x}}\left\langle\bar{J}\left(x_{\mathrm{s}}\right)\left(O_{\mathrm{s}}(x)-\left\langle O_{\mathrm{s}}(x)\right\rangle\right) J(0)\right\rangle}{\sum_{\mathbf{x}_{\mathbf{s}}, \mathbf{x}}\left\langle\bar{J}\left(x_{\mathrm{s}}\right)\left(O_{1}-\left\langle O_{1}(x)\right\rangle\right) J(0)\right\rangle},
$$

where $J$ is an operator with quantum numbers of the nucleon and $O_{1}=\bar{u} u+\bar{d} d$ and $O_{\mathrm{s}}=2 \bar{s} s$. The calculation of the ratio of Eq. (7) is particularly challenging because of very noisy contributions originating from disconnected diagrams. In Eq. (7), $x=(t, \mathbf{x})$ and $x_{s}=\left(t_{s}, \mathbf{x}_{\mathbf{s}}\right)$ denote the Euclidean time and space coordinates. We will refer to $t$ and $t_{s}$ as the source-operator separation and the source-sink separation, respectively. We have shown in [16] that $R\left(t, t_{s}\right)$ does not need to be renormalized since no mixing in the renormalization pattern appears. The ratio $R\left(t, t_{s}\right)$ has the following asymptotic behavior:

$$
R\left(t, t_{s}\right)=y_{N}+\mathcal{O}\left(e^{-\Delta M t}\right)+\mathcal{O}\left(e^{-\Delta M\left(t_{\mathrm{s}}-t\right)}\right)
$$

where we have denoted with $\Delta M$ the mass gap between the ground state and the first excited state of the nucleon. Note that the two additional contributions to $y_{N}$ are nonvanishing as long as $t$ and $t_{\mathrm{s}}$ are finite. These two contributions are a systematic effect inherent to any lattice calculation and will be referred to as excited state contamination in the following.

More details on our setup and on the technique to evaluate Eq. (7) can be found in $[16,18]$ where we discuss in particular the crucial points of our improved variance reduction technique and of the nonperturbative renormalization. 


\section{CHIRAL EXTRAPOLATION}

A crucial element in the determination of $y_{N}$ is the extrapolation to the physical value of the pion mass. Ideally, to this end chiral perturbation theory should be used. Let us therefore briefly sketch how the leading order chiral perturbation theory behavior of $y_{N}$ can be obtained.

Using the Feynman Hellman theorem, the sigma terms can be related to the derivative of the nucleon mass with respect to the light or strange quark masses:

$$
\sigma_{\pi N}=m_{l} \frac{\partial m_{N}}{\partial m_{l}}, \quad \sigma_{s}=m_{s} \frac{\partial m_{N}}{\partial m_{s}},
$$

and the $y_{N}$ parameter can be written as follows:

$$
y_{N}=2 \frac{\partial m_{N}}{\partial m_{s}}\left(\frac{\partial m_{\mathrm{PS}}^{2}}{\partial m_{l}} \frac{\partial m_{N}}{\partial m_{\mathrm{PS}}^{2}}\right)^{-1}
$$

where we have neglected the strange quark mass dependence of $m_{\mathrm{PS}}^{2}$.

The nucleon mass dependence on the pion mass can be described by the leading one-loop result in $S U(2)$ heavy baryon chiral perturbation theory $\mathrm{HB} \chi \mathrm{PT}$ [19] which reads

$$
m_{N}\left(m_{\mathrm{PS}}\right)=m_{N}^{(0)}-4 c^{(1)} m_{\mathrm{PS}}^{2}-\frac{3 g_{A}^{2}}{32 \pi f_{\pi}^{2}} m_{\mathrm{PS}}^{3}+\mathcal{O}\left(m_{\mathrm{PS}}^{4}\right) .
$$

Here $g_{A}$ is the axial coupling of the nucleon and $f_{\pi}$ is the pion decay constant. We take the physical value $g_{A}=$ 1.2695 and use the convention where the physical value of $f_{\pi}$ is $92.4 \mathrm{MeV}$. Note that the leading order expansion of the light sigma term reads $\sigma_{\pi N}=-4 c^{(1)} m_{\mathrm{PS}}^{2}$ and thus $c^{(1)}$ has to be strictly negative to give a positive value for $\sigma_{\pi N}$.

For the dependence of the nucleon strange sigma terms as a function of the pseudoscalar meson mass, we use the following Ansatz:

$$
\langle N|\bar{s} s| N\rangle=d_{0}+d_{1} m_{\mathrm{PS}}^{2}+\mathcal{O}\left(m_{\mathrm{PS}}^{3}\right)
$$

with $d_{0}, d_{1}$ as fit coefficients. Similar expressions can be derived also from EFT, see for instance [20].

Taking also the leading order chiral perturbation theory expression of the pseudoscalar meson mass $m_{\mathrm{PS}}^{2}=2 B m_{1}$ into account, the leading expression for the $y_{N}$ parameter reads

$$
y_{N}=y_{N}^{(0)}+y_{N}^{(1)} m_{\mathrm{PS}}+\mathcal{O}\left(m_{\mathrm{PS}}^{2}\right)
$$

where

$$
y_{N}^{(0)}=\frac{d_{0}}{-4 B c^{(1)}}, \quad y_{N}^{(1)}=\frac{9 d_{0} g_{A}^{2}}{2 B \pi\left(4 c^{(1)}\right)^{2} 32 f_{\pi}^{2}} .
$$

This simple leading order expression predicts that $y_{N}$ is an increasing function of $m_{\mathrm{PS}}$. As we will see in the following, this contradicts the behavior of our data for $y_{N}$. An interpretation of this mismatch is that higher orders of chiral perturbation theory would be needed to describe our results. However, since this implies further free fit parameters more data points than we have presently would be necessary to be able to apply such higher order expressions. In addition the validity of such a chiral expansion is questionable for pion masses above $300 \mathrm{MeV}$ (see e.g [21]) and thus cannot be applied to describe most of the lattice data presented here. This lack of being able to apply chiral perturbation theory led us to use simple linear and quadratic fit Ansätze in the pseudoscalar mass, as will be discussed below.

\section{RESULTS AND SYSTEMATIC EFFECTS}

Our results for $y_{N}$ are shown in Fig. 1 as a function of $m_{\mathrm{PS}}$. The chiral behavior of the $y_{N}$ parameter is a difficult issue and we are not aware of a direct computation for the quark mass dependence of $y_{N}$ itself in the framework of EFT. As discussed above, our set of data points is insufficient to apply higher order chiral perturbation theory. It turned out that the number of fit parameters is too large to obtain reliable fits and, with our data set, it was not possible to disentangle different orders of the chiral expansion.

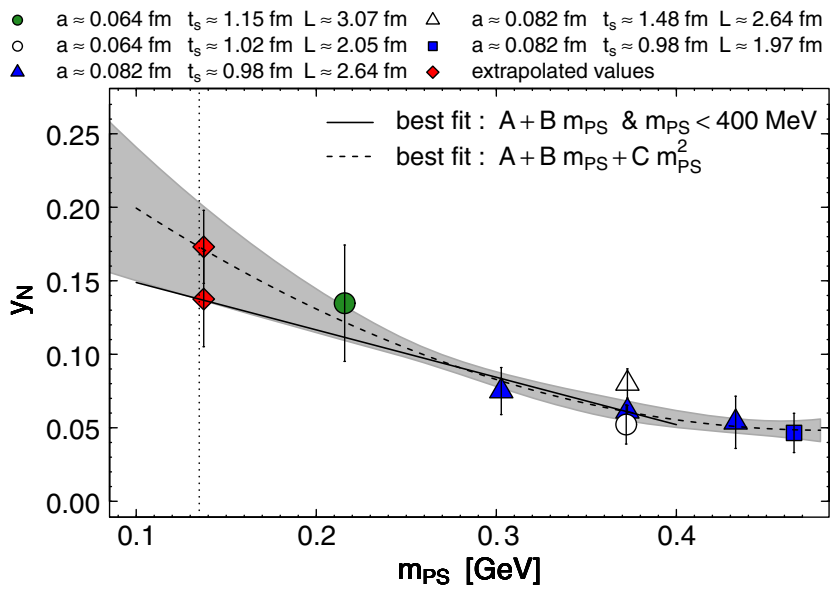

FIG. 1 (color online). Our results for $y_{N}$ as a function of $m_{\mathrm{PS}}$. The values of the lattice spacing $a$, the linear extent of the box $L$ and the source-sink separation $t_{\mathrm{s}}$ used here are given in the legend. We extrapolate to the physical value of the pion mass (marked by the vertical dotted line) using linear (solid line) and quadratic (dashed line) fits in $m_{\mathrm{PS}}$. For the quadratic fit, we also show the corresponding error band. Points represented by open symbols are only taken to estimate systematic effects and are not included in our final analysis. 
We therefore follow here the approach to use simple polynomial fit Ansätze for the pion mass dependence of $y_{N}$.

In the graph, we use different values of the lattice spacing $a$, the physical linear extent of the box $L$ and the sourcesink separation $t_{\mathrm{s}}$. We perform an extrapolation to the physical pion mass employing a linear fit in $m_{\mathrm{PS}}$ (solid line) and a quadratic one (dashed line). Note that only the points marked by filled symbols are included in the fits and that only data for $m_{\mathrm{PS}}<400 \mathrm{MeV}$ are included in the linear fit. Open symbols are solely used to demonstrate systematic effects which will be discussed in more detail below. The vertical dotted line in Fig. 1 marks the physical value of the pion mass.

In our Osterwalder-Seiler setup the value of the valence strange quark mass has been tuned in order to match the kaon mass obtained in the unitary setup. In principle, other matching conditions could also be used leading to different values of the valence strange quark mass. By computing $y_{N}$ for valence strange quark masses varying them by about $40 \%$ we could not detect any significant change in $y_{N}$ within our statistical error. Hence, below we will not consider the tuning of the valence strange quark mass as a source of systematic errors.

As mentioned above, the excited state's contamination needs to be scrutinized carefully in order to obtain reliable results. This is particularly delicate in the case of nucleon matrix elements because the statistical error grows exponentially when $t$ or $t_{s}$ are increased. Determining the asymptotic regime in $t$ and $t_{s}$ where the two last terms of Eq. (8) can be safely neglected and a clear plateau behavior appears is thus often difficult given the typical statistics of lattice calculations for nucleon observables. We therefore performed a detailed analysis of this effect on a single gauge ensemble increasing by more than 1 order of magnitude the statistics used.

We computed the ratio $R\left(t, t_{s}\right)$ for $t_{s} \sim 1.0 \mathrm{fm}$ (filled triangle in Fig. 1) and $t_{s} \sim 1.5 \mathrm{fm}$ (open triangle in Fig. 1) keeping the value of $a=0.08 \mathrm{fm}$ and $L=2.6 \mathrm{fm}$ fixed. We then performed several constant fits of the ratio $R\left(t, t_{s}\right)$ varying the fit interval $\left[t_{\min } / a, t_{\max } / a\right]$. We then chose the longest plateau such that the fit on a restricted range $\left[t_{\min } / a-1, t_{\max } / a-1\right]$ change marginally compared to the one obtained fitting on the range $\left[t_{\min } / a, t_{\max }\right]$. Since such a fitting window can be found we conclude that the systematic error introduced by the choice of a particular fitting range is negligible. We summarize the fitting range dependence for various source-sink separations in Table I. Note that the plateaus have a good quality because of a large cancellation of the $t$ dependence of the numerator and denominator in the ratio $R\left(t, t_{s}\right)$. In particular, we find $y_{N}=$ $0.061(4)$ for $t_{s}=1.0 \mathrm{fm}$ and $y_{N}=0.080(10)$ for $t_{s}=$ $1.5 \mathrm{fm}$ which indicates a non-negligible excited state's contamination of about $\sim 32 \%$. Note that we also performed computations for intermediate source-sink separations of 1.14 and $1.31 \mathrm{fm}$. The results are summarized in Table I and
TABLE I. Dependence on the fitting window of $y_{N}$ for various source-sink separations. The fitting range and the $\chi^{2} /$ ndof are indicated.

\begin{tabular}{lccr}
\hline \hline$t_{s}$ & {$\left[t_{\min } / a, t_{\max } / a\right]$} & $y_{N}$ & $\chi^{2} / \mathrm{ndof}$ \\
\hline $12 a \approx 0.98 \mathrm{fm}$ & {$[3,9]$} & $0.061(4)$ & $1.3 / 6$ \\
$12 a \approx 0.98 \mathrm{fm}$ & {$[4,8]$} & $0.061(4)$ & $0.4 / 4$ \\
$14 a \approx 1.15 \mathrm{fm}$ & {$[3,11]$} & $0.063(5)$ & $2.33 / 8$ \\
$14 a \approx 1.15 \mathrm{fm}$ & {$[4,10]$} & $0.064(5)$ & $1.38 / 6$ \\
$16 a \approx 1.31 \mathrm{fm}$ & {$[6,10]$} & $0.070(7)$ & $0.72 / 4$ \\
$16 a \approx 1.31 \mathrm{fm}$ & {$[7,9]$} & $0.071(7)$ & $0.17 / 2$ \\
$18 a \approx 1.48 \mathrm{fm}$ & {$[5,13]$} & $0.080(10)$ & $2.2 / 8$ \\
$18 a \approx 1.48 \mathrm{fm}$ & {$[6,12]$} & $0.082(10)$ & $1.01 / 6$ \\
\hline \hline
\end{tabular}

as can be seen the latter source-sink separation is compatible with the result obtained for a source-sink separation of $\sim 1.5 \mathrm{fm}$. The effect of different source-sink separations on our data is also exhibited in Fig. 2 where we show the ratio $R\left(t, t_{s}\right)$ for two source-sink separations of $0.98 \mathrm{fm}$ and $1.31 \mathrm{fm}$. Note also that in [16] we obtained a result at $t_{s}=1 \mathrm{fm}$ with a much lower statistic $\left[y_{N}=0.082(16)\right]$ which is fully compatible with the results quoted here. We thus consider as a conservative choice to use the difference between the two values for $y_{N}$ obtained at $t_{s}=1.0 \mathrm{fm}$ and $t_{s}=1.5 \mathrm{fm}$ as an estimate of our systematic error originating from the excited state's contamination assuming that this systematic effect does not depend strongly on the pseudoscalar meson mass. As an additional check we performed global fits of the ratio $R$ as a function of $t$ and $t_{s}$ for all the source-sink separations included in Table I. The asymptotic behavior of the ratio $R$ can be written as follows assuming that only one state contributes:

$$
R\left(t, t_{s}\right)=y_{N}+Z\left(e^{-\delta m t}+e^{-\delta m\left(t_{s}-t\right)}\right)
$$

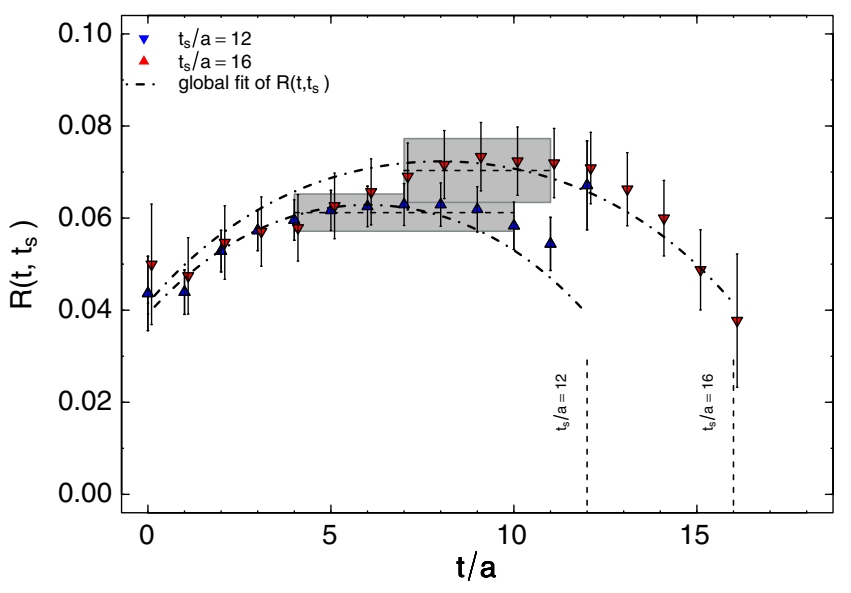

FIG. 2 (color online). $R\left(t, t_{s}\right)$ of Eq. (7) for two source-sink separations as a function of $t / a$. The source-sink separations are indicated by vertical dotted lines. The best plateau fits together with their statistical error are represented by gray bands. The best fit curves of the global fit of $R\left(t, t_{s}\right)$ are shown by black dotted curves. 
for a range of time parametrized by $t \geq t_{\text {cut }}$ and $t_{s}-t \leq t_{\text {cut }}$ and where $\delta m$ is the difference of the energy of the first excited state and the nucleon mass. The three coefficients $\left(y_{N}, Z, \delta m\right)$ are treated as free parameters. The best fit values for $t_{\text {cut }} / a=1 \mathrm{read}$

$$
\begin{aligned}
y_{N} & =0.091(7), \quad Z=0.047(5) \quad \text { and } \\
\delta m & =0.203(30)
\end{aligned}
$$

with $\chi^{2} /$ ndof $=23 / 53$. The corresponding curves are shown in Fig. 2 for $t_{s} / a=12$ and $t_{s} / a=16$. Estimating $y_{N}$ in this way thus gives a result compatible within statistical errors with the value obtained for $t_{s}=18 a$ quoted in Table I. The best fit value for $\delta m \approx 490(70) \mathrm{MeV}$ is larger than the mass of the pion on this ensemble, as expected for a nucleon-pion two particle state at nonzero momentum. We conclude that the difference between the results obtained at $t_{s} / a=18$ and $t_{s} / a=12$ gives a reasonable estimate of the systematic error due to the excited state contamination.

Note that for the other gauge ensembles used in Fig. 1, we have used the same procedure to determine the fitting range $\left[t_{\min } / a, t_{\max } / a\right]$ of the ratio $R\left(t, t_{s}\right)$ and also found a marginal dependence of the results though with larger statistical errors.

We have computed $y_{N}$ also at two different volumes (filled triangles and filled square in Fig. 1). However, we could not detect any significant finite volume effects within the statistical errors and thus finite volume effects can be safely neglected. We also show in Fig. 1 results for $y_{N}$ for two different lattice spacings (filled triangle and empty circle in Fig. 1). The two points are clearly compatible, indicating that lattice discretization effects are small. We took the difference between the values of these two data points as an estimate of the discretization errors.

In summary our final result reads

$$
y_{N}=0.173(29)(36)(19)(9)
$$

where the central value is given by the quadratic fit, the first error is statistical, and the last three errors are our estimates of systematic uncertainties, namely the chiral extrapolation, the excited state's contamination and the discretization error, respectively. Note that the systematic errors are substantially larger than the statistical one and therefore dominate the total error. Adding all errors in quadrature, we find $y_{N}=0.173(50)$.

\section{DISCUSSION}

There are a number of lattice works that concentrate on the determination of $\sigma_{s}$ using direct and/or indirect computation (see for instance [22-25] for $N_{f}=2+1$ results). Other lattice works for $N_{f}=2+1$ provide indirect determinations of $y_{N}$ [26-29] and our result for $y_{N}$ is in agreement with these works. We stress, however, that in

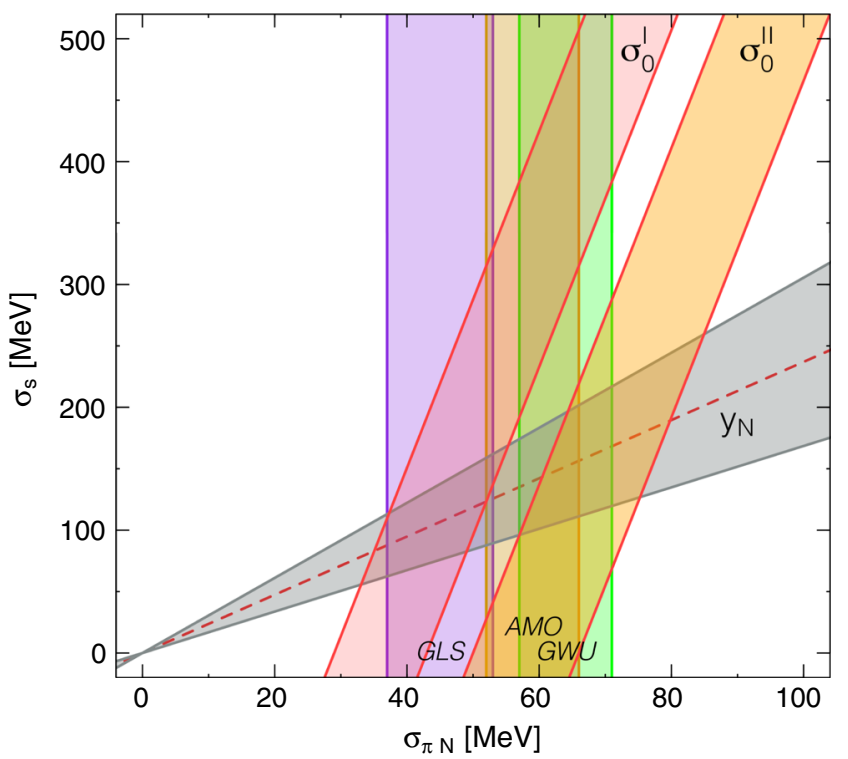

FIG. 3 (color online). Constraints on $\sigma_{s}$ obtained from our determination of $y_{N}$. The phenomenological determination of $\sigma_{\pi N}$ are represented by colored band as obtained from [10] (GLS), [11] (GWU) and [12] (AMO). We also show the constraint provided by the estimates $\sigma_{0}^{\mathrm{I}}$ [13] and $\sigma_{0}^{\mathrm{II}}[14]$. As can be seen the value of $y_{N}$ can constrain the value of $\sigma_{s}$ to be smaller than about $250 \mathrm{MeV}$.

our work we were able to perform a comprehensive analysis of systematic uncertainties covering lattice spacing and finite volume effects and, in particular, a careful investigation of excited state contamination. Furthermore computing directly the ratio of the matrix element $y_{N}$ allows us to avoid any assumptions on the domain of the validity of EFT relations which is based on $S U(2)$ or $S U(3)$ $\mathrm{HB} \chi \mathrm{PT}$ expansion and sometimes known only at leading order accuracy.

As in [30], we show in Fig. 3 the $\left(\sigma_{\pi N}, \sigma_{s}\right)$ plane together with vertical colored bands that represent the phenomenological determinations and the corresponding uncertainties of $\sigma_{\pi N}$ mentioned in the Introduction. In order to put further constraints on $\sigma_{s}$, we use the following relations, $\sigma_{s}=\frac{1}{2} \frac{m_{s}}{m_{l}}\left(\sigma_{\pi N}-\sigma_{0}\right)=y_{N} \frac{1}{2} \frac{m_{s}}{m_{l}} \sigma_{\pi N}$, together with the ratio of the quark masses taken from the FLAG group [31]. A first constraint derives from using the phenomenological determination of $\sigma_{0}$ (indicated by $\sigma_{0}$ in Fig. 3). The work performed here provides a constraint through our direct computation of $y_{N}$ (gray contour) which includes the estimate of both statistical and systematic errors. As can be seen the result constrains the strange $\sigma$ term and suggests an upper bound of $\approx 250 \mathrm{MeV}$ for $\sigma_{s}$.

\section{CONCLUSIONS AND OUTLOOK}

In this work, we have performed a direct computation of the strangeness of the nucleon $y_{N}$, including light, strange and charm sea quarks with an emphasis on the study of 
systematic effects. Using maximally twisted mass fermions which allow for an efficient noise reduction technique and which avoids mixing under renormalization we have obtained $y_{N}=0.173(50)$. Our result for $y_{N}$ is compatible with previous determinations [26-29,32] but includes an analysis of systematic errors originating from discretization, chiral extrapolation and excited states' uncertainties. It is worth pointing out that we find a rather low value of the strange $\sigma$ term with corresponding consequences for the nucleon-dark matter cross section.

One important conclusion of our work is that the error we obtain for $y_{N}$ is dominated by systematic uncertainties, in particular the chiral extrapolation and excited state contamination which cannot be neglected. While the error from the chiral extrapolation can be avoided in future calculations which are performed at or very close to the physical value of the pion mass, the excited state contamination must be carefully assessed. Thus, future lattice evaluations of the $y_{N}$ parameter using the physical value of the light quark mass will avoid the systematic error due to the chiral extrapolation, but they will still have to address excited state contamination, as this study has demonstrated.

\section{ACKNOWLEDGMENTS}

We thank our fellow members of ETMC for their constant collaboration. We are grateful to the John von Neumann Institute for Computing (NIC), the Jülich Supercomputing Center and the DESY Zeuthen Computing Center for their computing resources and support. This work has been supported in part by the DFG Sonderforschungsbereich/ Transregio SFB/TR9-03, by the Cyprus Research Promotion Foundation under Contract No. KY- $/ 0310 / 02 /$, and the Research Executive Agency of the European Union under Grant Agreement No. PITN-GA-2009-238353 (ITN STRONGnet). K. J. was supported in part by the Cyprus Research Promotion Foundation under Contract No. ПРО $\Sigma$ Е $\Lambda$ KY $\Sigma$ H/EMПЕIPO $\Sigma / 0311 / 16$.
[1] J. Gasser and H. Leutwyler, Phys. Rep. 87, 77 (1982).

[2] Z. Ahmed et al. (CDMS-II Collaboration), Phys. Rev. Lett. 106, 131302 (2011).

[3] R. Bernabei et al. (DAMA Collaboration), Eur. Phys. J. C 56, 333 (2008).

[4] C. Aalseth et al. (CoGeNT Collaboration), Phys. Rev. Lett. 106, 131301 (2011).

[5] T. Sumner et al. (ZEPLIN-III Collaboration), AIP Conf. Proc. 1200, 963 (2010).

[6] J. Jochum et al., Prog. Part. Nucl. Phys. 66, 202 (2011).

[7] J. Ellis and K. A. Olive, in Particle Dark Matter: Observations, Models and Searches, edited by G. Bertone (Cambridge University Press, Cambridge, England, 2013), p. 142.

[8] G. Servant and T. M. Tait, New J. Phys. 4, 99 (2002).

[9] G. Bertone, K. Kong, R. R. de Austri, and R. Trotta, Phys. Rev. D 83, 036008 (2011).

[10] J. Gasser, H. Leutwyler, and M. Sainio, Phys. Lett. B 253, 252 (1991).

[11] M. M. Pavan, I. I. Strakovsky, R. L. Workman, and R. A. Arndt, PiN Newsl. 16, 110 (2002).

[12] J. Alarcon, J. Martin Camalich, and J. Oller, Phys. Rev. D 85, 051503 (2012).

[13] B. Borasoy and U.-G. Meissner, Ann. Phys. (Berlin) 254, 192 (1997).

[14] J. M. Alarcon, L. S. Geng, J. Martin Camalich, and J. A. Oller, Phys. Lett. B 730, 342 (2014).

[15] Note that in [14], the authors obtain $y_{N}=0.02(13)(10)$ and $\sigma_{0}$ around $60 \mathrm{MeV}$.

[16] S. Dinter, V. Drach, R. Frezzotti, G. Herdoiza, K. Jansen, and G. Rossi (ETM Collaboration), J. High Energy Phys. 08 (2012) 037.
[17] R. Baron et al., J. High Energy Phys. 06 (2010) 111.

[18] P. Boucaud et al. (ETM Collaboration), Comput. Phys. Commun. 179, 695 (2008).

[19] J. Gasser, M. E. Sainio, and A. Svarc, Nucl. Phys. B307, 779 (1988).

[20] J.-W. Chen and M. J. Savage, Phys. Rev. D 66, 074509 (2002).

[21] S. Dürr et al. (Budapest-Marseille-Wuppertal Collaboration), Phys. Rev. D 90, 114504 (2014).

[22] D. Toussaint and W. Freeman (MILC Collaboration), Phys. Rev. Lett. 103, 122002 (2009).

[23] P. Junnarkar and A. Walker-Loud, Phys. Rev. D 87, 114510 (2013).

[24] H. Ohki, K. Takeda, S. Aoki, S. Hashimoto, T. Kaneko, H. Matsufuru, J. Noaki, and T. Onogi (JLQCD Collaboration), Phys. Rev. D 87, 034509 (2013).

[25] M. Gong et al., Phys. Rev. D 88, 014503 (2013).

[26] S. Durr et al., Phys. Rev. D 85, 014509 (2012).

[27] R. Horsley, Y. Nakamura, H. Perlt, D. Pleiter, P. E. L. Rakow, G. Schierholz, A. Schiller, H. Stüben, F. Winter, and J. M. Zanotti, Phys. Rev. D 85, 034506 (2012).

[28] R. Young and A. Thomas, Phys. Rev. D 81, 014503 (2010).

[29] P. Shanahan, A. Thomas, and R. Young, Phys. Rev. D 87, 074503 (2013).

[30] R. Young, Proc. Sci., LATTICE2012 (2012) 014 [arXiv:1301.1765].

[31] G. Colangelo et al., Eur. Phys. J. C 71, 1695 (2011).

[32] G. S. Bali et al. (QCDSF Collaboration), Phys. Rev. D 85, 054502 (2012). 